This document is confidential and is proprietary to the American Chemical Society and its authors. Do not copy or disclose without written permission. If you have received this item in error, notify the sender and delete all copies.

\title{
Solvent Sorption Induced Actuation of Polymer-Based Composites Based on a Polymer of Intrinsic Microporosity
}

\begin{tabular}{|r|l|}
\hline Journal: & ACS Applied Polymer Materials \\
\hline Manuscript ID & ap-2020-01215j.R2 \\
\hline Manuscript Type: & Article \\
\hline Date Submitted by the & 22-Dec-2020 \\
\hline Complete List of Authors: & $\begin{array}{l}\text { Polak-Kraśna, Katarzyna; University of Bath, Mechanical Engineering; } \\
\text { National University of Ireland Galway, College of Science and } \\
\text { Engineering } \\
\text { Tian, Mi; University of Bath, Chemical Engineering; University of Exeter, } \\
\text { College of Engineering, Mathematics and Physical Sciences } \\
\text { Rochat, Sébastien; University of Bath, Chemistry; University of Bristol, } \\
\text { School of Chemistry and Bristol Composites Institute } \\
\text { Gathercole, Nicholas; University of Bath, Mechanical Engineering } \\
\text { YUAN, CHENGGANG; University of Bath, Mechanical Engineering } \\
\text { Hao, Zhe; University of Bath, Mechanical Engineering } \\
\text { Pan, Min; University of Bath, Mechanical Engineering } \\
\text { Burrows, Andrew; University of Bath, Department of Chemistry } \\
\text { Mays, Timothy; University of Bath, Chemical Engineering } \\
\text { Bowen, Chris; University of Bath, Materials Research Centre }\end{array}$ \\
\hline & \\
\hline
\end{tabular}

\section{SCHOLARONE \\ Manuscripts}




\title{
Solvent Sorption Induced Actuation of Polymer-Based
}

\section{Composites Based on a Polymer of Intrinsic Microporosity}

\author{
Katarzyna Polak-Kraśna*a,b, Mi Tiann ${ }^{\mathrm{c}, \mathrm{d}}$, Sébastien Rochate, ${ }^{\mathrm{ef}}$, Nicholas Gathercole ${ }^{\mathrm{a}}$, Chenggang Yuan ${ }^{\mathrm{a}}$, \\ Zhe Hao ${ }^{\mathrm{a}}$, Min Pan ${ }^{\mathrm{a}}$, Andrew D. Burrows ${ }^{\mathrm{e}}$, Timothy J. Mays ${ }^{\mathrm{c}}$, Chris R. Bowen ${ }^{\mathrm{a}}$ \\ aDepartment of Mechanical Engineering, University of Bath, Claverton Down, Bath, BA2 7AY, United Kingdom \\ 'Department of Chemical Engineering, University of Bath, Claverton Down, Bath, BA2 7AY, United Kingdom \\ eDepartment of Chemistry, University of Bath, Claverton Down, Bath, BA2 7AY, United Kingdom \\ ${ }^{b}$ Biomedical Engineering, College of Science and Engineering, National University of Ireland, Galway, H91 TK33, Republic \\ of Ireland (present address) \\ ${ }^{\mathrm{d} C o l l e g e}$ of Engineering, Mathematics and Physical Sciences, University of Exeter, Exeter, EX4 4QF, United Kingdom \\ fSchool of Chemistry and Bristol Composites Institute (ACCIS), University of Bristol, Bristol, BS8 1TH, United Kingdom
}

*corresponding author: Dr. K. Polak-Kraśna, address: Alice Perry Building, National University of Ireland, Galway, H91 TK33 Galway, Republic of Ireland, e-mail address: polak.krasna@gmail.com 
Abstract

Materials that are capable of actuation in response to a variety of external stimuli are of significant interest for applications in sensors, soft robotics and biomedical devices. Here, we present a class of actuator using composites based on a polymer of intrinsic microporosity (PIM). By adding an activated carbon (AX21) filler to a PIM, the composite exhibits repeatable actuation upon solvent evaporation and wetting and it is possible to achieve highly controlled three-dimensional actuation. Curled composite actuators are shown to open upon exposure to a solvent and close as a result of solvent evaporation. The degree of curling and actuation is controlled by adjusting the amount of filler and evaporation rate of the solvent casting process, while the actuation speed is controlled by adjusting the type of solvent. The range of forces and actuation speed produced by the composite is demonstrated using acetone, ethanol and dimethyl sulfoxide as the solvent. The maximum contractile stress produced upon solvent desorption in the pure PIM polymer reached $12 \mathrm{MPa}$, with an ultimate force over 20,000 times the weight of a sample. This form of composite actuator is insensitive to humidity and water, which makes it applicable in an aqueous environment, and can survive a wide range of temperatures. These characteristics make it a promising actuator for the diverse range of operating conditions in robotic and medical applications. The mechanism of actuation is discussed, which is based on asymmetric distribution of the carbon filler particles that leads to a bilayer structure and the individual layers expand and contract differently in response of solvent wetting and evaporation, respectively. Finally, we demonstrate the application of the actuator as a potential drug delivery vehicle, with capacity for encapsulating two kinds of drugs and reduced drug leakage in comparison to existing technologies.

keywords: actuator, polymer composite, polymer of intrinsic microporosity, drug delivery, microorigami capsule 


\section{Introduction}

Polymers that are able to react to external stimuli by changing their properties have recently attracted interest due to their applications in biomedicine, mechanical actuation, sensing, soft robotics and selfhealing surfaces. Materials designed to change their shape in a controllable and reversible manner have been considered in the production of drug delivery systems ${ }^{1}$, gene delivery vehicles ${ }^{2}$, precision actuators and switches ${ }^{3-6}$, artificial muscles ${ }^{7}$, and walking devices ${ }^{8}$. Such adaptive soft matter can be classified depending on the triggering mechanism that initiates the shape-changing behaviour; this can include temperature ${ }^{9}$, electric charge ${ }^{3,7,10}$, humidity ${ }^{6,11-14}, \mathrm{pH}^{2}$, solvents ${ }^{13-16}$ or UV light ${ }^{17}$. The development of such new materials is often inspired by the strategies of living organisms to sense the environment or achieve actuation. As an example, plant systems such as pine cones are able to generate movement by a differential swelling of different parts of their tissue ${ }^{5}$. Similar mechanisms have been employed in hydrogels to achieve controlled and reversible changes in shape under conditions of changing humidity; however, their response time is usually slow (up to several hours) and the generated stress is low due to high water content ${ }^{18}$.

A variety of bilayer actuators have been fabricated that incorporate active layers on a passive substrate. For example, Ma et al. developed a multilayer film actuator capable of carrying significant loads ( 0.375 $\mathrm{mN} ; 128$ times actuator's weight) which was triggered by changes in humidity ${ }^{8}$. Their actuator exploited the different swelling rates of film layers, thereby generating internal strains. A singlematerial actuator based on gradient dewetting of water and other solvents was presented by Wu et al. ${ }^{16}$, where a fast curling response was achieved based on gradient swelling of the material. Zhao et al. developed a hygroscopic actuator capable of bending in response to acetone vapor and humidity by introducing a gradient of porosity through the membrane thickness ${ }^{14}$. The authors demonstrated that the graded porous structure can yield high forces $(0.75 \mathrm{mN} ; 25$ times actuator's weight) as a result of a gradient desorption mechanism. A limitation of humidity powered devices is their susceptibility to external conditions, thereby requiring a controlled environment. The difficulty in achieving precise control, due to humidity fluctuations, and a slow response time remain significant challenges in 
successful implementation of humidity-driven actuators. In addition, many existing polymer actuators are susceptible to humidity and extreme temperatures and require complex manufacturing methodology. Soft actuators with high contractile force provide a significant advantage in manufacturing soft robots for diverse engineering use. The high contractile force provided by the solvent-driven materials enable the roboticists to design robots with larger deformation and more flexible segment bodies, which provides the opportunities for new robot design without impairing the static and dynamic responses of the robots.

As a result, in order to develop an efficient and universal soft actuator, there is a need to create actuator materials that are easily manufactured, are capable of rapid and reversible deformation, can deliver high loads and are able to withstand a wide range of hot/cold temperatures and humidity levels.

Here we present a composite based on a microporous polymer to create actuators that exhibit rapid, reversible and repeatable deformation that is accompanied by very high loads in the presence of a solvent (ethanol, acetone, and DMSO), but remain insensitive to water and humidity, and can withstand a large temperature range. The actuator is formed from a polymer of intrinsic microporosity, PIM-1 which exhibits strong shrinkage in presence of a solvent. PIM-1 is a processable polymer with a unique combination of properties, which includes a high surface area $\left(\sim 800 \mathrm{~m}^{2} \mathrm{~g}^{-1}\right)$ and good solvent processability due to its rigid, but kinked, backbone structure which prevents efficient space packing of the polymer chains. This leads to the creation of nanoscale pores smaller than $2 \mathrm{~nm}$ in three dimensions 19. While the intrinsic microporosity of these materials has attracted significant interest for applications related to separation membranes ${ }^{20}$ and gas storage ${ }^{21-23}$, we demonstrate in this paper that these intriguing materials can be successfully used as actuators and drug delivery vehicles. To achieve threedimensional actuation, PIM-1 is doped with an activated carbon filler, AX21 (Anderson Development Co., Adrian, MI, US). It was prepared from a mixture of $\mathrm{KOH}$ and petroleum coke by activation at 700 ${ }^{\circ} \mathrm{C}$, the surface area is in the range of $2800-3500 \mathrm{~m}^{2} \mathrm{~g}^{-1} 24$. As a result of the asymmetric distribution of filler in the composite, a bilayer structure if formed where each layer produces a different internal strain in response to a solvent. We investigate the influence of solvent type, of the evaporation speed and of the filler content on the magnitude and kinetics of actuation. We present their applicability after being 


\section{Materials and methods}

\subsection{Polymer and composite samples preparation}

PIM-1 was synthesized using the method described previously ${ }^{21}$. To summarise, 5,5',6,6'tetrahydroxy-3,3,3',3'-tetramethyl-1,10-spirobisindane $\quad\left(\begin{array}{llllll}5.11 & \mathrm{~g}, & 15 & \mathrm{mmol}, & 1 & \text { eq. }) \text {, }\end{array}\right.$ tetrafluoroterephthalonitrile (3.00 g, $15 \mathrm{mmol}, 1$ eq.) and anhydrous $\mathrm{K}_{2} \mathrm{CO}_{3}(16.59 \mathrm{~g}, 120 \mathrm{mmol}, 8 \mathrm{eq}$. were using as starting materials. The solids were evacuated and backfilled with nitrogen three times and $100 \mathrm{ml}$ of anhydrous DMF was added, after which precipitation was achieved while stirring for three days at $65{ }^{\circ} \mathrm{C}$ under $\mathrm{N}_{2}$. The solution was then hydrated, filtered, air-dried, and purified via reprecipitation. The final step was dissolving PIM-1 in chloroform and re-precipitating with $\mathrm{MeOH}$ three times before drying under vacuum at $80{ }^{\circ} \mathrm{C}$ overnight.

The number-average molar mass $\left(M_{n}\right)$ of the obtained PIM-1 measured with Gel Permeation Chromatography (GPC) in the previous study was $76,261 \mathrm{~g} / \mathrm{mol}$, weight-average molar mass $\left(M_{w}\right)$ was $193,074 \mathrm{~g} / \mathrm{mol}$, and dispersity index $\oslash=2.5^{21}$.

Polymer films were cast by dissolving PIM-1 (1.22 g) in chloroform $(35 \mathrm{~mL})$ and pouring the solution into a large $200 \mathrm{~mm}$ Petri dish, where it was left to evaporate for $48 \mathrm{~h}$. To prepare PIM-1/AX21 composite films, the activated carbon AX21 was separately stirred with a small amount of chloroform ( $5 \mathrm{~mL}$ ) for $24 \mathrm{~h}$ and added to the PIM-1 solution. The mixture was then stirred for another $24 \mathrm{~h}$ at room temperature. Different ratios of AX21:PIM-1 mixtures were prepared to obtain 10, 20, 30, 40 and 50 wt $\%$ of AX21 in the PIM-1 composites. Solutions were poured into $200 \mathrm{~mm}$ Petri dishes and left to evaporate for 48 hours. The evaporation speed was optimized to achieve mechanically robust films and to tailor the distribution of filler and actuation capacity. To assess the effect of evaporation speed, composite films with $40 \mathrm{wt} \%$ concentration of AX21 filler were evaporated in an open container for 12 hours, in a semi-closed container for several days and in a closed container for a period of 2 weeks. After solvent casting, all films were dried under vacuum at $80{ }^{\circ} \mathrm{C}$ for $8 \mathrm{~h}$ to eliminate solvent residues. 
For testing purposes, rectangular samples were cut according to BS EN ISO 527-3:1996 standard (1/2 of Specimen type 2) ${ }^{25}$. Preliminary testing was used to confirm that the decreased dimensions of specimens did not influence the mechanical characterization results. Samples thickness was assessed using a Mitutoyo 227-211 Absolute Digimatic Micrometer (Mitutoyo, Kawasaki, Japan) with \pm 0.001 $\mathrm{mm}$ measurement accuracy and measuring force adjustment.

\subsection{Imaging of composite samples}

PIM-1/AX21 composite materials with different concentrations of AX21 in PIM-1 (10, 20, 30, 40 and $50 \mathrm{wt} \%$ ) were imaged using a Scanning Electron Microscope (SEM) (JSM6480LV, JEOL Ltd., Tokyo, Japan). In addition, $30 \mathrm{wt} \%$ samples manufactured with faster and slower evaporation rates (48 hours and two weeks) were compared to understand the influence of changes in the composite micro-structure due to the speed of evaporation and its impact on actuation behaviour; in particular the distribution of the AX21 particles in the composite. Cross-sections of the composite samples were obtained by brittle fracture of composite samples; they were then gold coated, and images of fracture surfaces were acquired.

\subsection{Contraction and actuation force measurement}

To assess the response of pure and dry PIM-1 films to the solvents used for actuation, the dimensions of pure PIM-1 samples were measured, and samples were immersed in ethanol and measured again after evaporation to determine the shrinking magnitude and its repeatability. PIM-1 contractile forces in response to a range of solvents evaporation were measured in tension. Rectangular samples were exposed to three different solvents; ethanol, propan-2-one (acetone) and dimethyl sulfoxide (DMSO, Sigma Aldrich, St. Louis, USA). Following solvent immersion for 1 minute, the samples were clamped at a fixed gauge length of $40 \mathrm{~mm}$ in a uniaxial tensile testing machine; an Instron 3369 instrument with a $50 \mathrm{~N}$ static load cell (Instron, Norwood, USA). An initial preload of $0.1 \mathrm{~N}$ was applied to produce a uniform tension along the sample. Contractile forces produced upon solvent evaporation were measured in the static setup using the load cell with no external strain applied. The force as a function of time was 
monitored to evaluate the rate of sample shrinkage with time. Three samples were tested with each solvent.

To measure the contractile forces and the influence of AX21 additive concentration on actuation force of the PIM-1/AX21 composites, composites with 10, 20, 30, 40 and $50 \mathrm{wt} \%$ of AX21 in PIM-1 were tested in the same tensile testing setup using ethanol as a solvent, and the force as a function of time was recorded. At least five samples were tested for each actuator composition.

\subsection{Thermal stability of PIM-1 and PIM-1/AX21 composites}

The ability of the composites to withstand hostile cold and hot conditions was also evaluated. PIM-1 samples and composite samples were subjected to a thermal treatment of $250{ }^{\circ} \mathrm{C}$ for 30 minutes. After heat treatment, they were exposed to ethanol and their swelling and shrinking behaviour was monitored. Samples were also exposed to liquid nitrogen for 30 seconds, then they were left to warm to room temperature and their actuation behaviour was evaluated.

\section{Results}

\subsection{Shrinkage of pure PIM-1 and autonomous actuation of PIM-1/AX21 composites}

Pure PIM-1 samples did not exhibit significant actuation prior to solvent treatment; they were entirely transparent, yellow in colour, and flat. After solvent wetting, they would simply swell, and then shrink upon solvent evaporation, with no out of plane bending or curling of the material. The sample length decreased as a result of ethanol evaporation by almost $10 \%$ of the original length; $100 \mathrm{~mm}$ to $91 \mathrm{~mm}$.

The composite samples became increasingly black in colour with a higher concentration of activated carbon in the polymer PIM-1 matrix. Samples with $10-20 \mathrm{wt} \%$ were yellow and partially transparent with clearly visible activated carbon particles distributed within the yellow polymer matrix. Samples with $30 \mathrm{wt} \%$ and higher filler content were almost entirely black and opaque. The bottom surface that 
was in contact with the glass Petri dish during casting was smooth and matte, while the top surface was rough and glossy in appearance, see Figure 2a. In samples with higher proportions of AX21, the apparent surface roughness increased. When freshly cast composite films were cut into rectangular strips they would spontaneously curl into loose helices or half-tubes, depending on the direction of the incision (longitudinal vs transverse) such as in Figure 2a. After solvent exposure they would return to a flat shape (Figure 2b) and on subsequent evaporation, the composites would spontaneously curl into tight tubes around 1-2 $\mathrm{mm}$ in diameter (Figure 2c,d). The described behaviour was observed in all composites starting from $20 \mathrm{wt} \%$ concentration of AX21 filler, up to $50 \mathrm{wt} \%$. Samples containing 10 wt\% AX21 in PIM-1 exhibited a lower degree of curling and formed loose helices rather than tubes. These films exhibited autonomous shape changing when exposed to a solvent which was reversible and repeatable.
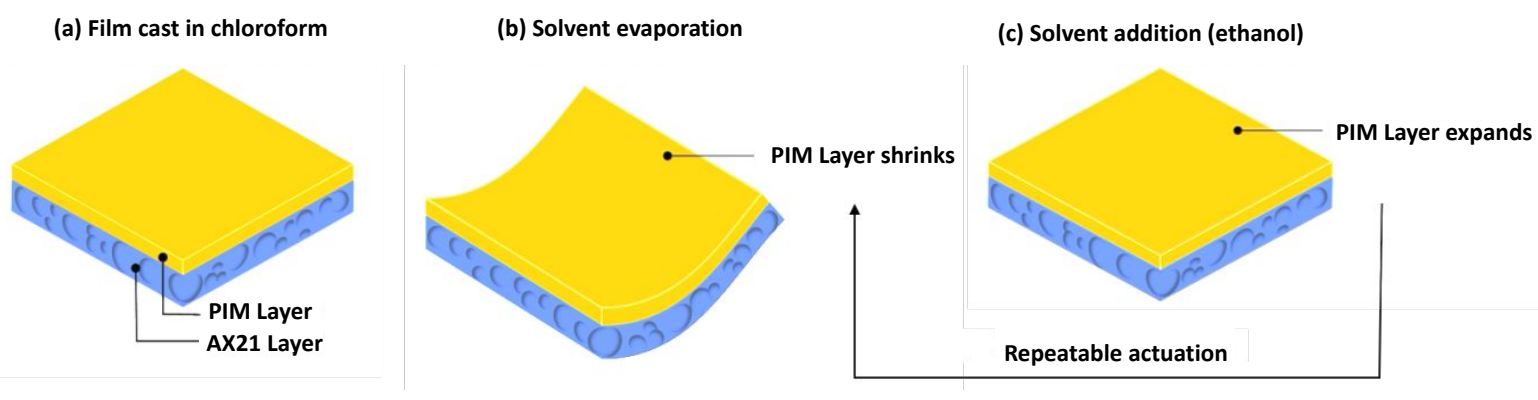

Figure 1 Repeatable actuation mechanism in PIM-1/AX21 composite. (a) Flat samples after casting in chloroform, (b) Curling occurring after solvent evaporation, (c) Film flattening upon wetting with solvent.

Curling occurred in such manner that the upper rough surface of a film was always the internal surface of the curled material, and the smooth surface was the outer surface. The curling behaviour could be reversed by moistening samples with solvent, as shown schematically in Figure 1. Upon wetting or soaking in a solvent, samples straightened into flat strips, Figure $2 \mathrm{~b}$. Upon solvent evaporation, the samples then exhibited rapid curling into tubes or helices, depending on their initial shape, see Figure 2c,d,f,g. The initial shape, as well as the direction of curling after solvent evaporation was dependent upon the direction of cutting during samples preparation. Transverse cutting caused the samples to curl 


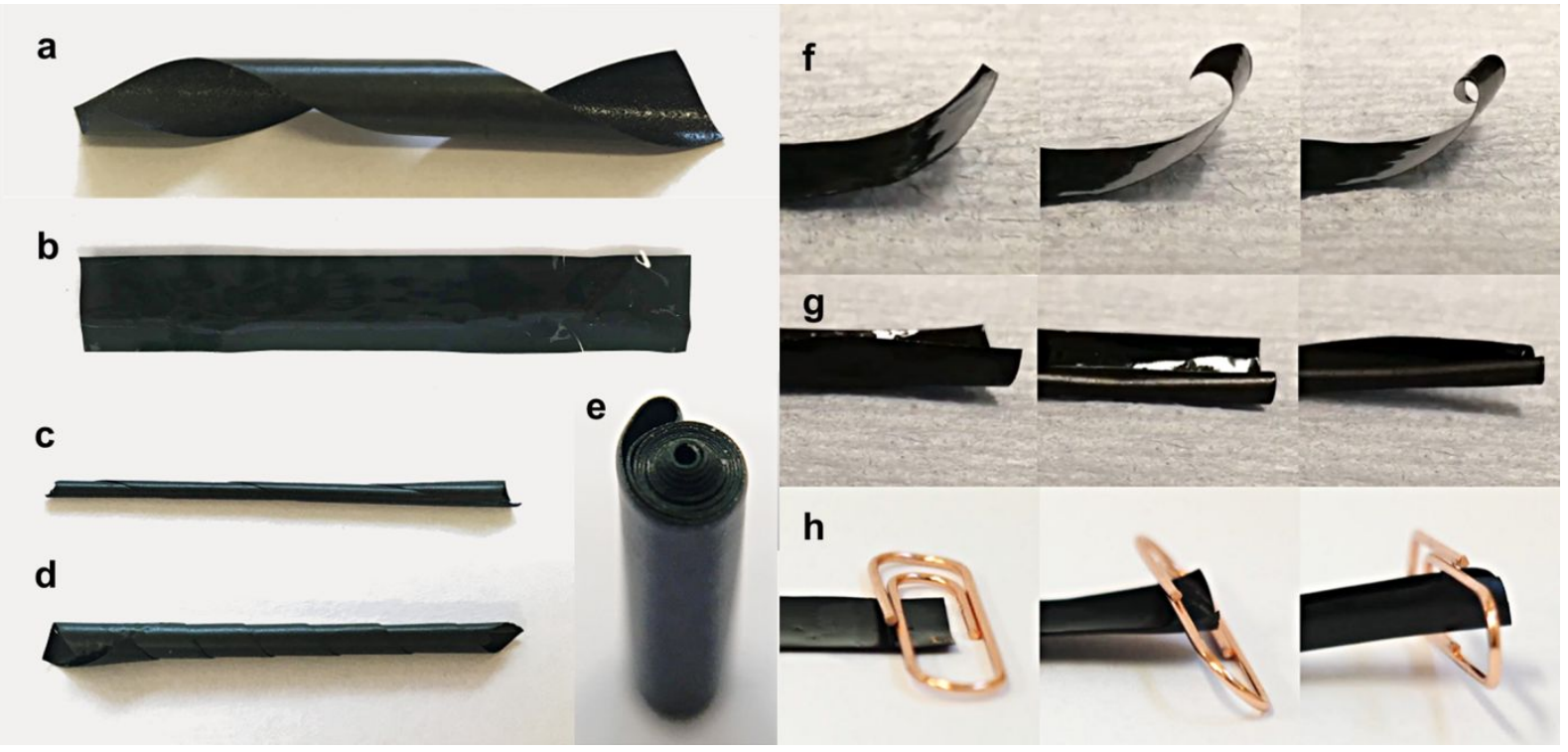

Figure 2 Different modes of autonomous curling and imposed curling in PIM-1/AX21 composites. All samples presented in the figure contained $40 \mathrm{wt} \%$ filler. Dimensions of all the samples in the relaxed state were $10 \mathrm{~mm}$ width and $75 \mathrm{~mm}$ length. All samples were obtained with 48 hours casting evaporation process. Solvent used was ethanol. (a) Sample before ethanol treatment exhibiting helical curling after a oblique incision, (b) Wet sample immersed in ethanol, (c) Dry sample autonomously 
curled along its long axis, d) Dry sample autonomously curled along its short axis, (e) Dry sample which was forced to curl into a tight tube and maintained the shape after drying, (f) Sample autonomously curling along its short axis during ethanol desorption after a transverse incision, (g) Sample autonomously curling along its long axis during ethanol desorption after a longitudinal incision, (h) Sample lifting an object over 10 times heavier than itself during autonomous actuation caused by ethanol desorption.

\subsection{Imaging of PIM/AX21 composite samples}

Micro-structural analysis of composite samples with different filler concentrations and samples evaporated for 48 hours or two weeks was performed by SEM, see Figure 3. Samples cast and evaporated over 48 hours (Figure 3, (s)) exhibited a distinct bi-layer structure with a clear interface between the lower porous and upper dense regions. This structure would form as the activated carbon filler would settle to the bottom of a film during evaporation, and a dense matrix that was characteristic of PIM-1 would be visible at the top of the film. The ratio of the porous filler-rich and filler-poor regions was dependent on the filler content in the composite, and the porous layer thickness would increase with an increase in concentration of AX21 filler, as indicated by the orange arrows in Figure 3. The distinction between two layers was less apparent in samples that were cast and evaporated over a longer period of two weeks (Figure 3, (1)) and their distribution was much more uniform and disorderly. This difference in structure had an impact of actuation/curling response and will be discussed later in relation to the actuation mechanism. 

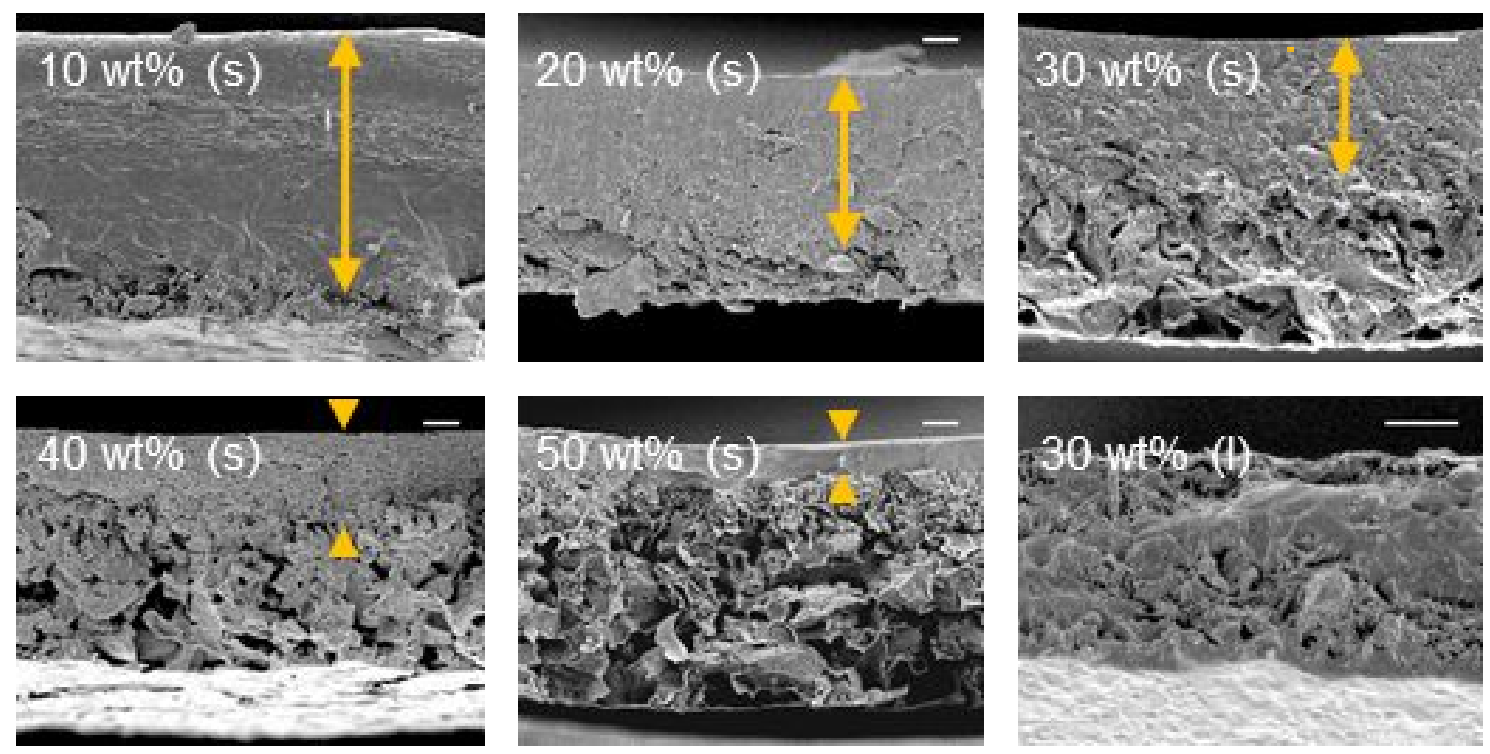

Figure 3 SEM images of cross-sections of PIM-1/AX21 composite samples cast and evaporated over 48 hours (s) with different filler concentrations and a $30 \mathrm{wt} \%$ sample cast and evaporated over two weeks (l), the scale bar in all images is $10 \mu \mathrm{m}$ in length. Yellow arrows point out the regions with topographies typical of pure PIM-1.

\subsection{Measurement of shrinkage and contractile forces}

The kinetics and magnitude of shrinking was dependent upon the type of solvent used and was assessed as a contractile force measurement of PIM-1 exposed to ethanol, acetone and DMSO, as shown in Figure 4. The maximum force was obtained for samples that had been immersed in acetone $(7.8 \mathrm{~N})$. Average force for samples exposed to acetone was almost $7 \mathrm{~N}$, whereas for ethanol it was 4.5 N. DMSO had lowest influence on contractile force as samples produced only $1 \mathrm{~N}$ upon DMSO evaporation. When the magnitude of maximum average load obtained with each type of solvent is plotted against solvent vapour pressure at $20^{\circ} \mathrm{C}$, as in Figure 5, it can be seen that the measured maximum force increased with an increase of vapour pressure. Acetone and ethanol both required $100 \mathrm{~s}$ to achieve maximum force which was higher for acetone. Using DMSO, the maximum value of force was achieved after over $400 \mathrm{~s}$. 


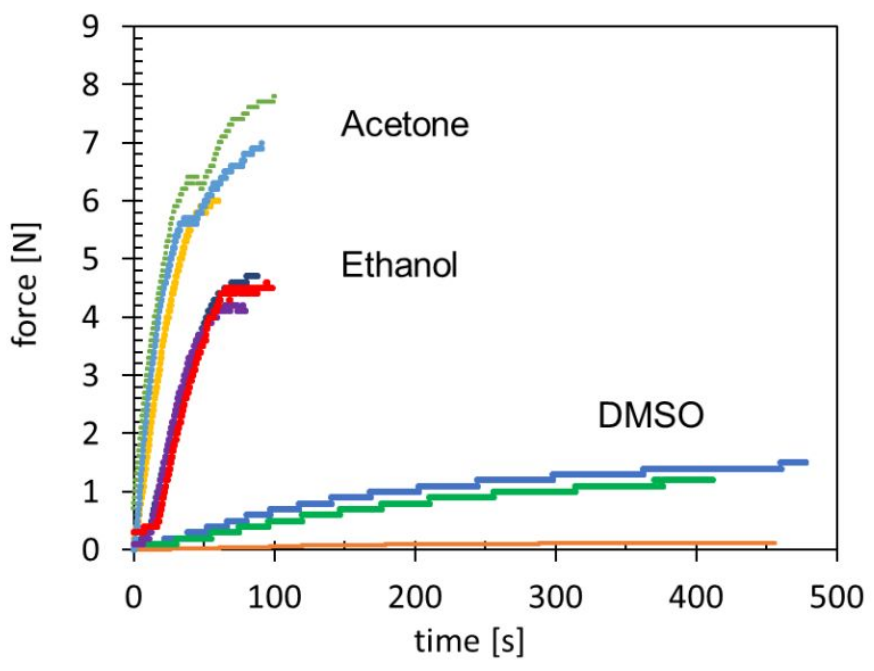

Figure 4 Contractile force of pure PIM-1 upon exposure and evaporation of different solvents.

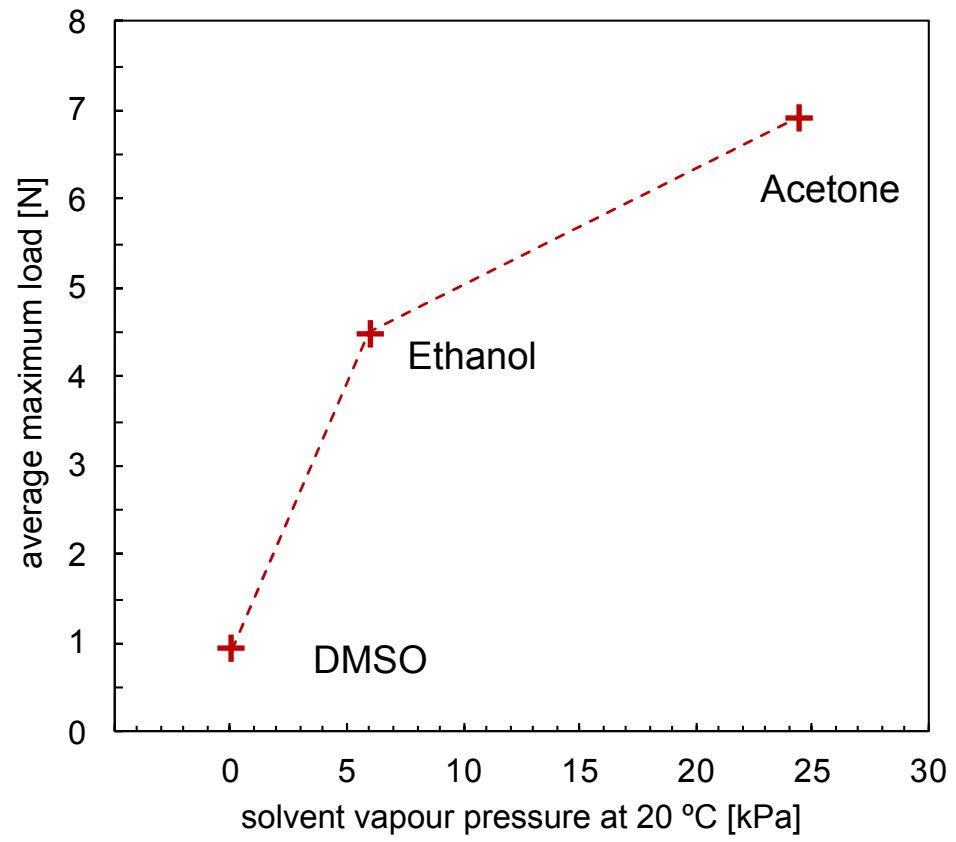

Figure 5 Average maximum load obtained with different solvents vs solvent vapour pressure at $20^{\circ} \mathrm{C}$.

Contractile forces measured in pure PIM-1 and PIM-1/AX21 composites upon solvent desorption in some cases were sufficient to cause mechanical failure of the materials if they were constrained in the mechanical test machine. Pure PIM-1 samples produced a stress of up to $12 \mathrm{MPa}$, associated with solvent evaporation. The highest ultimate force and stress for composite samples exposed to ethanol 


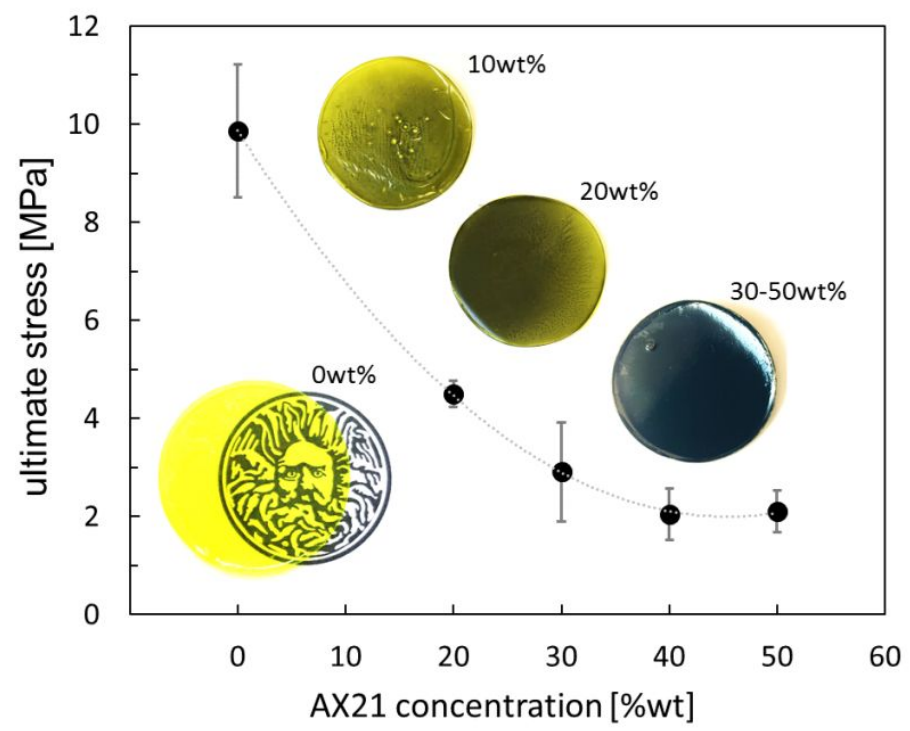

Figure 6 Average ultimate stress obtained from spontaneous contractile force measured for different concentrations of AX21 in PIM-1 with error bars representing standard deviations. The curve is provided to guide the eye only. Insets: (bottom left corner) Pure PIM-1 is perfectly transparent, (right top corner from top to bottom): Representative images of PIM-1/AX21 samples with different concentrations; $10 \mathrm{wt} \%, 20 \mathrm{wt} \%$, and 30-50 wt\% of AX21 in PIM-1. Samples with 30 wt\% and more AX21 were uniformly black.

\subsection{Thermal stability of PIM-1 and PIM-1/AX21 composites}

PIM-1 samples removed from the oven at temperature $250{ }^{\circ} \mathrm{C}$ remained mechanically robust and exhibited the same swelling and shrinking behaviour. Composite samples did not exhibit any changes in actuating behaviour after the same thermal treatment (see Supplementary Video 2). After treatment with liquid nitrogen, no degradation in actuation behaviour was observed (see Supplementary Video 
3) and samples subjected to both heating and liquid nitrogen treatment also maintained their actuation capabilities (see Supplementary Video 4).

\section{Discussion}

PIM-1 exhibits swelling and shrinking behaviour upon exposure to solvents (ethanol, acetone and DMSO), but remains insensitive to water. Shrinking after ethanol exposure led to a $12 \%$ decrease in sample length and forces with magnitude over 20,000 times the weight of the sample which is higher than previously reported $6,8,11,12,14$. While pure PIM-1 did not exhibit out of plane bending, the PIM1/AX21 composites exhibit tubular curling behaviour and have the capacity to be formed into desired shapes (Figure 2e), where the behaviour is reversible and repeatable. The demonstrated phenomenon can be of use for a number of applications in the fields of actuation, sensing, soft robotics, and artificial muscles. Three-dimensional actuation was specific for PIM-1/AX21 composites and was not observed in pure PIM-1 films, or composites with another filler such as fine particles of porous aromatic framework PAF-1 22 .

A similar mechanism of swelling-induced autonomous motility was previously observed in response to humidity ${ }^{6,12,14}$. The curling was dependent from the direction of sample incision as previously observed by Douezan et al. ${ }^{26}$. However, many actuators that exhibit solvent-induced actuation are also triggered by moisture and humidity ${ }^{14-16}$ and are therefore susceptible to changes in environmental conditions and are applicable only in controlled conditions. Zhao and co-workers developed a concept for a humidity sensitive membrane consisting of cationic poly(ionic liquid) polymer with gradient of porosity that bends in the presence of acetone vapor ${ }^{14}$. The membrane bends into a loop when placed in acetone vapor and quickly recovers its initial flat shape when back in air. The process we demonstrated here is opposite - the curled shape is the default state of the dry actuator (Figure 2c,d) and the relaxation of an actuator occurs upon exposure to an organic solvent (Figure 2b). Such a process is particularly attractive for utilising in delivery applications where a substance is enclosed inside a polymer tube and needs to be released upon solvent exposure. Additionally, the speed of the release can be controlled by adjusting the type of a solvent as the actuation kinetics vary for DMSO, acetone and ethanol. The difference in 
curling speed can be attributed to varying boiling points $\left(189{ }^{\circ} \mathrm{C}, 56^{\circ} \mathrm{C}\right.$ and $78{ }^{\circ} \mathrm{C}$, respectively) and volatility of the used solvents. A higher vapour pressure solvent evaporates faster, which leads to a rapid increase in the contractile force that causes more dynamic actuation. The magnitude of actuation has shown to be solvent vapour pressure dependent, therefore, could be controlled by adjusting solvent parameters. Therefore, the proposed solvent-driven actuator is programmable which enables controllable process of drug delivery as one of potential application areas with release upon solvent local application.

Multiple aspects of curling behaviour of PIM-1/AX21 actuators are highly controllable and can be programmed. The direction of curling can be controlled by the direction of incision during sample preparation process. Transverse cutting leads to curling along its short axis, longitudinal cutting produced samples curling along their long axis into tubes and oblique incision induce combined curling behaviour and the formation of helices. The magnitude of curling depends on the evaporation length during the solvent casting procedure. A slower evaporation of two weeks led to lower magnitudes of curling, as compared to higher magnitude after fast evaporation (48 hours). The contractile forces produced by the actuators are dependent on the amount of AX21 filler inside the PIM-1 matrix and the vapour pressure characteristics of a triggering solvent used. The solvent type also influences the speed of actuation.

\subsection{Actuation mechanism}

Our actuator materials use microporous polymer composites to obtain robust actuators with large actuation capacity. The curling behaviour of the composites can be explained by the presence of a gradient of carbon particles through the sample thickness, see Figure 3; it is noted that curling did not occur in pure PIM-1 or in our previous work on PIM-1 composites with homogenous distribution of a filler ${ }^{22}$. A porosity gradient has been shown to lead to bending behaviour in polymer composite membranes to an asymmetry of strain ${ }^{14}$. The gradient in our PIM-1/AX21 composites are formed due to range of particle sizes of the activated carbon filler and during the solvent casting-evaporation process, the larger carbon particles fall to the bottom of the chloroform solution, thus creating a 
bilaminar film structure in the case of rapid evaporation after casting, see Figure 3. We propose that the lower carbon-rich layer swells and shrinks to a lesser extent than the upper PIM-1-rich layer, (Figure 1) and this is in agreement with the observation that the lower smooth layer would always form the outer surface of the tube and the upper layer would form the inner layer of tube. It is also in agreement with the pure PIM-1 creating the largest forces on shrinkage when constrained in a tensile test machine, Figure 6. Finally, composites formed by slow evaporation after casting have exhibited a more even distribution of carbon, see Figure $2 \mathrm{f}$ and produce a smaller degree of curling; the more homogeneous distribution of filler may be a result of rearrangement of polymer chains and carbon particles in the solution due to slowly increasing density. The evaporation rate during casting can therefore be employed to control the curling magnitude. Additionally, the curvature of bending had been described to scale inversely with membrane thickness ${ }^{14}$ which can be considered another parameter enabling full control over an actuator's curling behaviour.

PIM-1 has shown no sensitivity to water due to its strong hydrophobic nature, which explains why it interacts differently with organic solvents than with water. As PIM-1 does not exhibit swelling in water, it is clear that organic solvents have stronger interactions with the polymer when compared to water which results in enhanced swelling behaviour when using ethanol, acetone, or DMSO.

Another mechanism of interest was reported by Ma et al. who found that a pentaerythritol ethoxylatepolypyrrole (PEE-PPy) composite increased its elastic modulus between moist and dry states, which they explained by the polymer structure being weakened upon water sorption, which recovered upon desorption ${ }^{6}$. This mechanism could explain the decreasing brittleness of PIM-1 films and composites when soaked in ethanol.

\subsection{Comparison and unique properties of PIM-1/AX21 actuators}

Zhao and co-workers measured a maximum force of $0.75 \mathrm{mN}$ produced by their actuator, which was 25 times the weight of the $3 \mathrm{mg}$ actuator ${ }^{14}$. Zhang described a moisture-responsive graphene oxide based actuator that could lift objects 50 times heavier ${ }^{15}$. The water gradient driven composite actuator presented by Ma et al. has shown an impressive capacity of lifting objects 380 times the weight of the actuator ${ }^{6}$. In this work, the maximum load achieved during contractile force measurement of pure PIM- 
1 was $7.8 \mathrm{~N}$ which is an equivalent of over 20,000 times the weight of a sample ( $35 \mathrm{mg})$. The maximum load achieved for a PIM-1/AX21 composite actuator reached $3.18 \mathrm{~N}$ constituting 9,000 times the weight of the sample. Both values are much higher than previously reported in the literature for humidity and solvent responsive actuators $6,8,11,12,14$. Actuators with such strength can be used as soft robotics joints in the development of walking devices, and the continuous swelling and contraction of the polymer could be employed to drive a piezoelectric element to power low-power devices via energy harvesting. High contractile force solvent-driven actuator in drug delivery applications would be able to carry more substance to the target locations.

The demonstrated actuator development methodology is applicable to composites with other types of additives that could introduce additional functionalities, whereas the simple solution processability enables employing other fabrication technologies such as electrospinning or 3D printing of structures with complex combined functionalities.

It has been shown that porosity can increase accessibility of solvents and promote mass transfer ${ }^{27}$. We propose that the high contractile forces achieved by the presented actuators are possible due to the microporous characteristics of the components and the combined high surface area of the composite which facilitates access and efficient mass transfer of the organic solvents within the structure and can enhance the kinematics and magnitude of actuation. Further analysis of how porosity, pore size and shape influence the kinematics of the solvent-driven actuator can provide insights into optimisation of the actuator and further possible applications.

What distinguishes our proposed actuator from other existing concepts $6,8,11,12,14$ is its stability in water, a feature necessary for artificial muscles or drug delivery vehicles to be applied in wet or humid environments. As an example, Zhao et al. report that their actuator can be triggered by combination of solvent vapour and humidity ${ }^{14}$, whereas the PIM-1/AX21 actuator responds only to organic solvents (ethanol, acetone and DMSO) while remaining inactive in water. As it cannot be triggered by humidity or addition of water, it can be placed in humid environment or in water while still being able to be triggered on demand (by solvent addition) as opposed to uncontrollable environmental changes. One of proposed applications for this type of material is in sensing the presence of a solvent and its concentration in a water solution. In addition, we have previously shown that PIM-1 alone has excellent 
thermal stability, does not become brittle in liquid nitrogen and is stable between $-150{ }^{\circ} \mathrm{C}$ and $350{ }^{\circ} \mathrm{C}$ 21; the PIM-1/AX21 composites start decomposing at $\sim 350{ }^{\circ} \mathrm{C}{ }^{23}$. In this study, we have demonstrated resistance to thermal treatment of PIM-1 and PIM-1/AX21 composites and that their actuating behaviour remains unchanged after heat treatment at $250{ }^{\circ} \mathrm{C}$ for 30 minutes and treatment with liquid nitrogen $\left(-196^{\circ} \mathrm{C}\right)$, which provides a wide range of operational temperatures. This excellent thermal stability makes the actuators suitable for applications in high temperature environment or for high temperature treatment such as steam sterilization necessary for medical applications. Their mechanical stability in low temperatures allows for long-term storage of micro-origami capsules loaded with drugs prior to application.

\section{Micro-origami capsule demonstration}

We now develop a demonstration for a proposed application of the actuator material as a drug delivery micro-origami capsule (see Figure 7 and Supplementary Video 5), where the external diameter of the capsule was $1.5 \mathrm{~mm}$ and the capsule length $10 \mathrm{~mm}$. A $40 \mathrm{wt} \%$ PIM-1/AX21 composite sample was used in the demonstration due to it high degree of actuation. Two different particle types (to represent potential drugs A and B) were loaded into the uncurled composite actuator after it was immersed in ethanol at room temperature (Figure 7 (i)) and then encapsulated autonomously along with the desorption of ethanol, Figure 7 (ii). The capsule can carry two different types of drugs which need to be separated from each other. After the completion of encapsulation, the actuator was delivered via water-powered driven flow to the target site, Figure 7 (iii). When the capsule reached the targeted site, ethanol was applied to open the micro-origami capsule for release, Figure 7 (iv). The corresponding folding curvature of the capsule versus ethanol desorption time is shown in Figure 8, where the whole process excluding delivery takes $22 \mathrm{~s}$. The capsule is fully curled from both ends naturally with two separated enclosed chambers (external diameter $=1.5 \mathrm{~mm}$, capsule length $=10 \mathrm{~mm}$ ). Larger or smaller capsule can be fabricated for various drug delivery applications. 
(i)

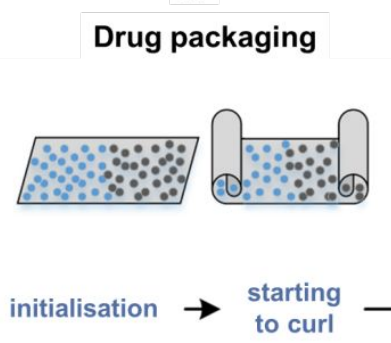

(ii)

Encapsulation

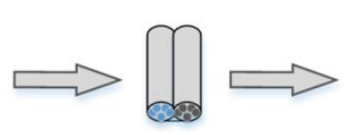

(iii)

Delivering

C. Delivering

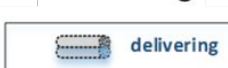

(iv)

\section{Sustained drug release}

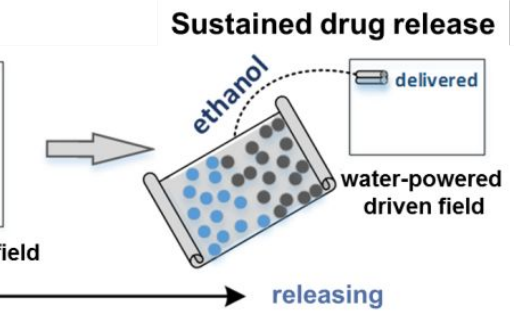

\section{- Molecular drug A \\ - Molecular drug B}

Figure 7 Schematic of micro-origami capsule for drug encapsulation and delivery. The capsule can carry two different types of drugs at the same time because of the advantages of the composite actuator which can naturally form two curled ends. The water-powered driven field is used for capsule delivery due its insensitivity to humidity and water.

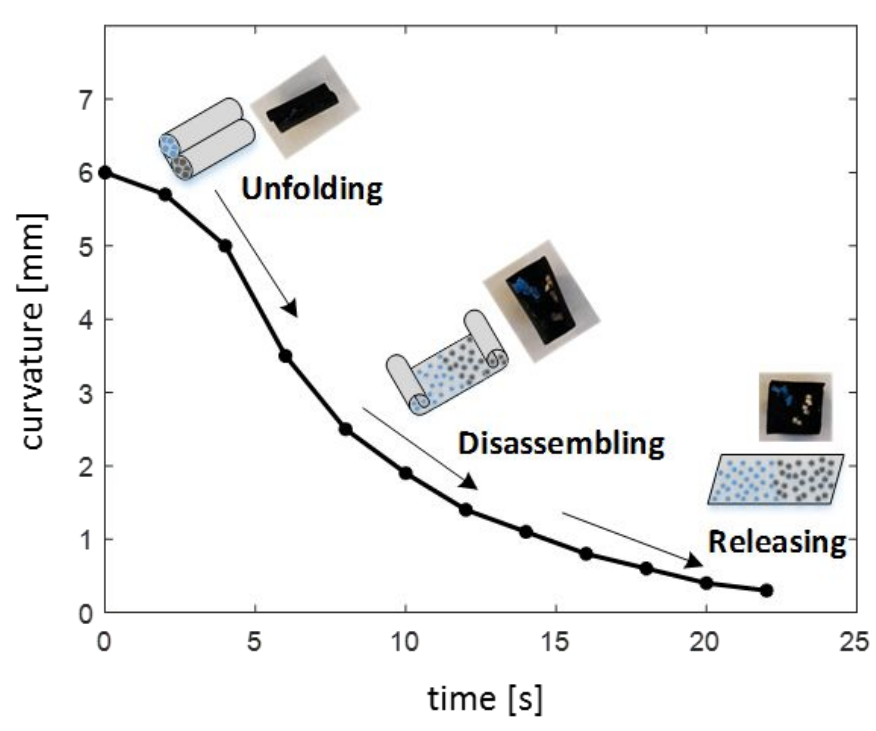

Figure 8 Curvature of the capsule versus ethanol desorption time.

The capsule is water resistant, and thus, the water-powered flow can effectively deliver the capsule to the target site, as shown in Figure 9. The delivery speed is determined by the power flow which can be adjusted for various requirements. 

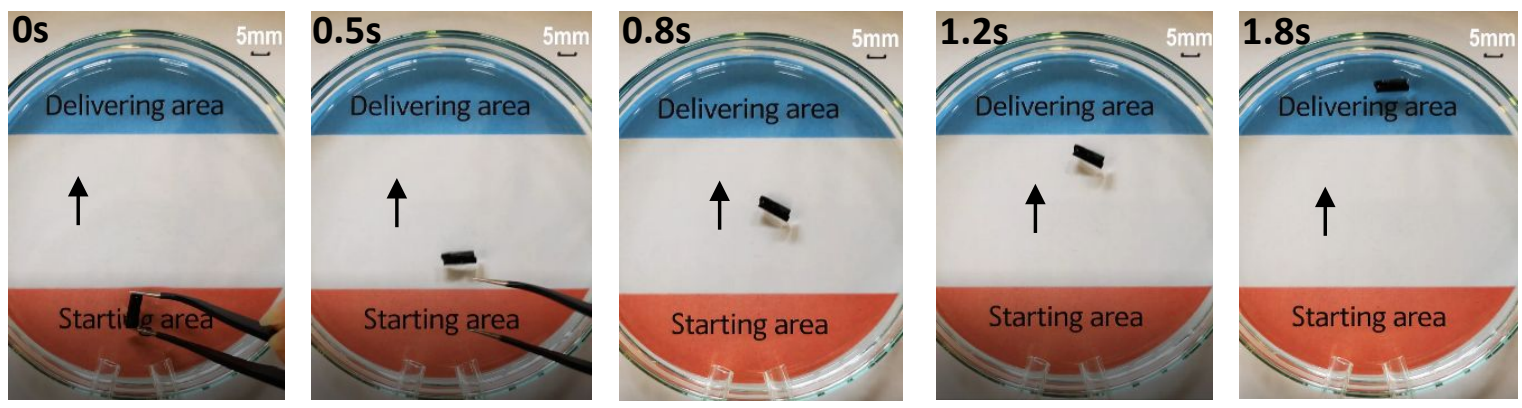

Figure 9 Capsule delivering using a water-powered driven field.

\section{Conclusions}

We have presented a class of composite polymer-based actuators and an approach to produce actuators that can be controlled by the presence of solvents. The magnitude and speed of actuation can be controlled by adjusting the amount of activated carbon filler added to the polymer matrix, the rate of solvent evaporation and the nature of the solvent. We have shown the highest reported contractile forces observed in polymer actuators triggered by a solvent other than water. Due to its insensitivity to water and humidity and its stability to high and low temperatures, the actuator can be applied in a range of environments and could be used as a solvent sensor. The inverse contractile mechanism in dry air makes the material of interest as a controlled delivery and release vehicle. We have demonstrated that the proposed actuator can be used as a micro-origami capsule for drug delivery, where the actuator could enclose two types of drugs, deliver them in water environment and release on demand in a desired location. The demonstration showed potential for significant advantages, such as eliminating drug leakage compared to current technologies.

\section{Conflict of interest statement}

The authors declare no competing financial interest. 


\section{Acknowledgements}

This work was financially supported by the UK Engineering and Physical Sciences Research Council (EPSRC) via SUPERGEN Grants EP/K021109/1 and EP/L018365/1.

\section{Supporting Information}

Supplementary Video 1 PIM-1/AX21 composite actuation. The video shows curling of PIM-1/AX21 sample with 40wt\% of AX21 in PIM-1 into a tight tube during ethanol desorption. Speed increased 8fold.

Supplementary Video 2 Actuator behaviour after heat treatment at $250^{\circ} \mathrm{C}$ for 30 minutes remains unchanged. Presented on the example of application as a drug delivery vehicle. Speed increased 32fold.

Supplementary Video 3 Actuator behaviour after immersing in liquid nitrogen remains unchanged. Presented on the example of application as a drug delivery vehicle. Speed increased 32-fold.

Supplementary Video 4 Actuator behaviour after heat treatment and liquid nitrogen treatment remains unchanged. Presented on the example of application as a drug delivery vehicle. Speed increased 32fold.

Supplementary Video 5 Drug delivery demonstration. Actuator is loaded with two kinds of drugs; it encapsulates the drugs in two separate chambers during ethanol desorption. Delivery of the capsule to the application site is achieved via water-powered driven field. After delivery, the micro-origami capsule is opened with ethanol and the drugs are released. 


\section{References}

(1) Caldorera-Moore, M. E.; Liechty, W. B.; Peppas, N. A. Responsive Theranostic Systems: Integration of Diagnostic Imaging Agents and Responsive Controlled Release Drug Delivery Carriers. Acc. Chem. Res. 2011, 44 (10), 1061-1070. https://doi.org/10.1021/ar2001777.

(2) Benns, J. M.; Choi, J. S.; Mahato, R. I.; Park, J. S.; Kim, S. W. PH-Sensitive Cationic Polymer Gene Delivery Vehicle: N-Ac-Poly(L-Histidine)-Graft-Poly(L-Lysine) Comb Shaped Polymer. Bioconjug. Chem. 2000, 11 (5), 637-645. https://doi.org/10.1021/bc0000177.

(3) Smela, E. Conjugated Polymer Actuators for Biomedical Applications. Adv. Mater. 2003, 15 (6), 481-494. https://doi.org/10.1002/adma.200390113.

(4) Smela, E. Conjugated Polymer Actuators. MRS Bull. 2008, 33 (3), 197-204. https://doi.org/10.1557/mrs2008.45.

(5) Fratzl, P.; Barth, F. G. Biomaterial Systems for Mechanosensing and Actuation. Nature 2009, 462 (7272), 442-448. https://doi.org/10.1038/nature08603.

(6) Ma, M.; Guo, L.; Anderson, D. G.; Langer, R. Bio-Inspired Polymer Composite Actuator and Generator Driven by Water Gradients. Science (80-. ). 2013, 339 (6116), 186-189. https://doi.org/10.1126/science.1230262.

(7) Must, I.; Kaasik, F.; Põldsalu, I.; Mihkels, L.; Johanson, U.; Punning, A.; Aabloo, A. Ionic and Capacitive Artificial Muscle for Biomimetic Soft Robotics. Adv. Eng. Mater. 2015, 17 (1), 84 94. https://doi.org/10.1002/adem.201400246.

(8) Ma, Y.; Zhang, Y.; Wu, B.; Sun, W.; Li, Z.; Sun, J. Polyelectrolyte Multilayer Films for Building Energetic Walking Devices. Angew. Chemie - Int. Ed. 2011, 50 (28), 6254-6257. https://doi.org/10.1002/anie.201101054.

(9) Hu, Z.; Zhang, X.; Li, Y. Synthesis and Application of Modulated Polymer Gels. Science (80-. ). 1995, 269 (5223), 525-527. https://doi.org/10.1126/science.269.5223.525.

(10) Sun, Z.; Yang, L.; Zhang, D.; Song, W. High Performance, Flexible and Renewable NanoBiocomposite Artificial Muscle Based on Mesoporous Cellulose/ Ionic Liquid Electrolyte 
Membrane. Sensors Actuators, B Chem. 2019, 283 (June 2018), 579-589. https://doi.org/10.1016/j.snb.2018.12.073.

(11) Zhang, Y.; Jiang, H.; Li, F.; Xia, Y.; Lei, Y.; Jin, X.; Zhang, G.; Li, H. Graphene Oxide Based Moisture-Responsive Biomimetic Film Actuators with Nacre-like Layered Structures. J. Mater. Chem. A 2017, 5 (28), 14604-14610. https://doi.org/10.1039/c7ta04208f.

(12) Zhang, L.; Liang, H.; Jacob, J.; Naumov, P. Photogated Humidity-Driven Motility. Nat. Commun. 2015, 6 (May), 1-12. https://doi.org/10.1038/ncomms8429.

(13) Zhang, K.; Geissler, A.; Standhardt, M.; Mehlhase, S.; Gallei, M.; Chen, L.; Thiele, C. M. Moisture-Responsive Films of Cellulose Stearoyl Esters Showing Reversible Shape Transitions. Sci. Rep. 2015, 5, 1-13. https://doi.org/10.1038/srep11011.

(14) Zhao, Q.; Dunlop, J. W. C.; Qiu, X.; Huang, F.; Zhang, Z.; Heyda, J.; Dzubiella, J.; Antonietti, M.; Yuan, J. An Instant Multi-Responsive Porous Polymer Actuator Driven by Solvent Molecule Sorption. Nat. Commun. 2014, 5, 4293-4301. https://doi.org/10.1038/ncomms5293.

(15) Liu, J. C.; Shang, Y. Y.; Zhang, D. J.; Xie, Z.; Hu, R. X.; Wang, J. X. Single-Material SolventSensitive Fluorescent Actuator from Carbon Dots Inverse Opals Based on Gradient Dewetting. Chinese J. Polym. Sci. (English Ed. 2017, 35 (9), 1043-1050. https://doi.org/10.1007/s10118017-1981-y.

(16) Wu, H.; Kuang, M.; Cui, L.; Tian, D.; Wang, M.; Luan, G.; Wang, J.; Jiang, L. Single-Material Solvent-Sensitive Actuator from Poly(Ionic Liquid) Inverse Opals Based on Gradient Dewetting. Chem. Commun. 2016, 52 (35), 5924-5927. https://doi.org/10.1039/c6cc01442a.

(17) Qin, C.; Feng, Y.; Luo, W.; Cao, C.; Hu, W.; Feng, W. A Supramolecular Assembly of CrossLinked Azobenzene/Polymers for a High-Performance Light-Driven Actuator. J. Mater. Chem. A 2015, 3 (32), 16453-16460. https://doi.org/10.1039/C5TA01543J.

(18) Ionov, L. Hydrogel-Based Actuators: Possibilities and Limitations. Materials Today. Elsevier December 1, 2014, pp 494-503. https://doi.org/10.1016/j.mattod.2014.07.002.

(19) Budd, P. M.; Ghanem, B. S.; Makhseed, S.; McKeown, N. B.; Msayib, K. J.; Tattershall, C. E. Polymers of Intrinsic Microporosity (PIMs): Robust, Solution-Processable, Organic Nanoporous Materials. Chem. Commun. 2004, 4 (2), 230-231. 
https://doi.org/10.1039/b311764b.

(20) McKeown, N. B. Polymers of Intrinsic Microporosity. ISRN Mater. Sci. 2012, 2012, 1-16. https://doi.org/10.5402/2012/513986.

(21) Polak-Kraśna, K.; Dawson, R.; Holyfield, L. T.; Bowen, C. R.; Burrows, A. D.; Mays, T. J. Mechanical Characterisation of Polymer of Intrinsic Microporosity PIM-1 for Hydrogen Storage Applications. J. Mater. Sci. 2017, 52 (7), 3862-3875. https://doi.org/10.1007/s10853-016-06474.

(22) Rochat, S.; Polak-Kraśna, K.; Tian, M.; Holyfield, L. T.; Mays, T. J.; Bowen, C. R.; Burrows, A. D. Hydrogen Storage in Polymer-Based Processable Microporous Composites. J. Mater. Chem. A 2017, 5 (35), 18752-18761. https://doi.org/10.1039/c7ta05232d.

(23) Tian, M.; Rochat, S.; Polak-Kraśna, K.; Holyfield, L. T.; Burrows, A. D.; Bowen, C. R.; Mays, T. J. Nanoporous Polymer-Based Composites for Enhanced Hydrogen Storage. Adsorption 2019, 25 (4), 889-901. https://doi.org/10.1007/s10450-019-00065-х.

(24) Rouquerol, J.; Rouquerol, F.; Llewellyn, P.; Maurin, G.; Sing, K. S. W. Adsorption by Powders and Porous Solids: Principles, Methodology and Applications: Second Edition; Elsevier: Amsterdam, 2013. https://doi.org/10.1016/C2010-0-66232-8.

(25) ISO. ISO 527-3: 2018 Plastics — Determination of Tensile Properties — Test Conditions for Films and Sheets. 2018.

(26) Douezan, S.; Wyart, M.; Brochard-Wyart, F.; Cuvelier, D. Curling Instability Induced by Swelling. Soft Matter 2011, 7 (4), 1506-1511. https://doi.org/10.1039/c0sm00189a.

(27) Lee, J. S. M.; Cooper, A. I. Advances in Conjugated Microporous Polymers. Chem. Rev. 2020, 120 (4), 2171-2214. https://doi.org/10.1021/acs.chemrev.9b00399.

\section{List of Figures}

Figure 1 Repeatable actuation mechanism in PIM-1/AX21 composite. (a) Flat samples after casting in chloroform, (b) Curling occurring after solvent evaporation, (c) Film flattening upon wetting with solvent. .......8

Figure 2 Different modes of autonomous curling and imposed curling in PIM-1/AX21 composites. All samples presented in the figure contained $40 \mathrm{wt} \%$ filler. Dimensions of all the samples in the relaxed state were $10 \mathrm{~mm}$ width and $75 \mathrm{~mm}$ length. All samples were obtained with 48 hours casting evaporation process. Solvent used was ethanol. (a) Sample before ethanol treatment exhibiting helical curling after a oblique incision, (b) Wet 
sample immersed in ethanol, (c) Dry sample autonomously curled along its long axis, d) Dry sample autonomously curled along its short axis, (e) Dry sample which was forced to curl into a tight tube and maintained the shape after drying, (f) Sample autonomously curling along its short axis during ethanol desorption after a transverse incision, (g) Sample autonomously curling along its long axis during ethanol desorption after a longitudinal incision, (h) Sample lifting an object over 10 times heavier than itself during autonomous actuation caused by ethanol desorption.

Figure 3 SEM images of cross-sections of PIM-1/AX21 composite samples cast and evaporated over 48 hours (s) with different filler concentrations and a $30 \mathrm{wt} \%$ sample cast and evaporated over two weeks (l), the scale bar in all images is $10 \mu \mathrm{m}$ in length. Yellow arrows point out the regions with topographies typical of pure PIM1 .

Figure 4 Contractile force of pure PIM-1 upon exposure and evaporation of different solvents.

Figure 5 Average maximum load obtained with different solvents vs solvent vapour pressure at $20^{\circ} \mathrm{C}$.....

Figure 6 Average ultimate stress obtained from spontaneous contractile force measured for different concentrations of AX21 in PIM-1 with error bars representing standard deviations. The curve is provided to guide the eye only. Insets: (bottom left corner) Pure PIM-1 is perfectly transparent, (right top corner from top to bottom): Representative images of PIM-1/AX21 samples with different concentrations; $10 \mathrm{wt} \%$, $20 \mathrm{wt} \%$, and 30$50 \mathrm{wt} \%$ of AX21 in PIM-1. Samples with $30 \mathrm{wt} \%$ and more AX21 were uniformly black.

Figure 7 Schematic of micro-origami capsule for drug encapsulation and delivery. The capsule can carry two different types of drugs at the same time because of the advantages of the new composite actuator which can naturally form two curled ends. The water-powered driven field is used for capsule delivery due its insensitivity to humidity and water.

Figure 8 Curvature of the capsule versus ethanol desorption time. .19

Figure 9 Capsule delivering using a water-powered driven field. 
solvent
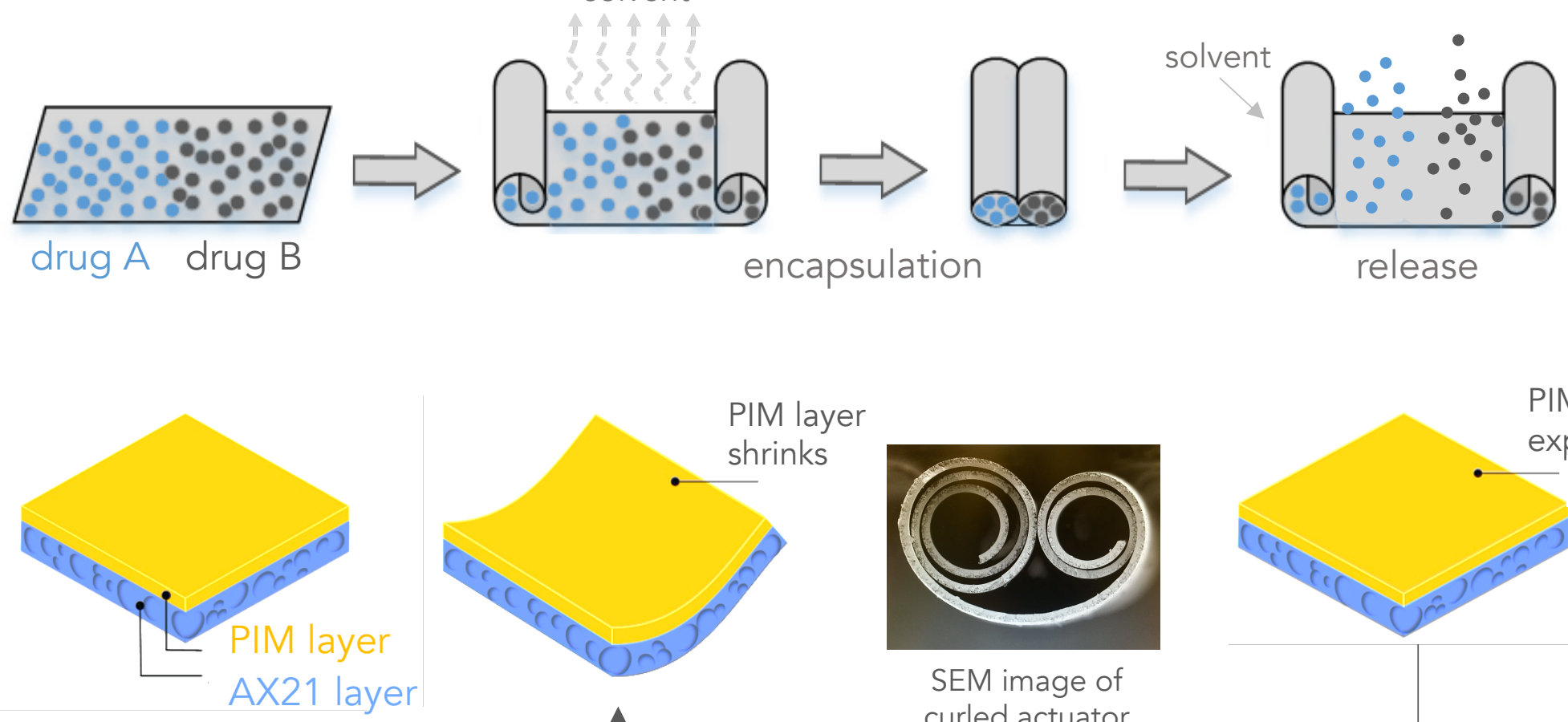

PIM layer

expands

,

SEM image of curled actuator

ACS Paragon Plus lepeatable actuation

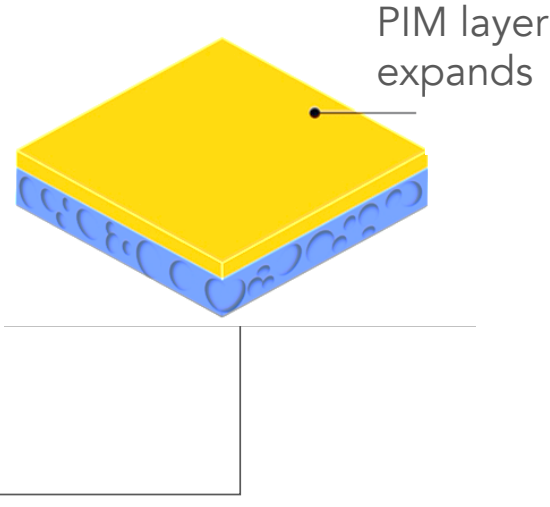

25

26

27 